\title{
GENERALIZED CORE INVERSES OF MATRICES
}

\author{
SANZHANG XU, JIANLONG CHEN, JULIO BENÍTEZ, AND DINGGUO WANG
}

Received 12 April, 2018

\begin{abstract}
In this paper, we introduce two new generalized inverses of matrices, namely, the $\langle i, m\rangle$-core inverse and the $(j, m)$-core inverse. The $\langle i, m\rangle$-core inverse of a complex matrix extends the notions of the core inverse defined by Baksalary and Trenkler [1] and the core-EP inverse defined by Manjunatha Prasad and Mohana [10]. The $(j, m)$-core inverse of a complex matrix extends the notions of the core inverse and the DMP-inverse defined by Malik and Thome [9]. Moreover, the formulae and properties of these two new concepts are investigated by using matrix decompositions and matrix powers.
\end{abstract}

2010 Mathematics Subject Classification: 15A09; 15A23

Keywords: $\langle i, m\rangle$-core inverse, $(j, m)$-core inverse, core inverse, DMP-inverse, core-EP inverse

\section{INTRODUCTION}

Let $\mathbb{C}^{m \times n}$ denote the set of all $m \times n$ complex matrices. Let $A^{*}, \mathcal{R}(A)$ and $\operatorname{rk}(A)$ denote the conjugate transpose, column space, and rank of $A \in \mathbb{C}^{m \times n}$, respectively. For $A \in \mathbb{C}^{m \times n}$, if $X \in \mathbb{C}^{n \times m}$ satisfies $A X A=A, X A X=X,(A X)^{*}=A X$, and $(X A)^{*}=X A$, then $X$ is called a Moore-Penrose inverse of $A$. This matrix $X$ is unique and denoted by $A^{\dagger}$. A matrix $X \in \mathbb{C}^{n \times m}$ is called an outer inverse of $A$ if it satisfies $X A X=X$; is called a $\{2,3\}$-inverse of $A$ if it satisfies $X A X=$ $X$ and $(A X)^{*}=A X$; is called a $\{1,3\}$-inverse of $A$ if it satisfies $A X A=A$ and $(A X)^{*}=A X$; is called a $\{1,2,3\}$-inverse of $A$ if it satisfies $A X A=A, X A X=X$ and $(A X)^{*}=A X$.

The core inverse of a complex matrix was introduced by Baksalary and Trenkler [1]. Let $A \in \mathbb{C}^{n \times n}$. A matrix $X \in \mathbb{C}^{n \times n}$ is called a core inverse of $A$, if it satisfies $A X=P_{A}$ and $\mathcal{R}(X) \subseteq \mathcal{R}(A)$, here $P_{A}$ denotes the orthogonal projector onto $\mathcal{R}(A)$. If such a matrix exists, then it is unique and denoted by $A^{\boxplus}$. For a square complex matrix $A$, one has that $A$ is core invertible, $A$ is group invertible, and $\operatorname{rk}(A)=\operatorname{rk}\left(A^{2}\right)$ are three equivalent conditions (see [2]). We denote $\mathbb{C}_{n}^{C M}=\left\{A \in \mathbb{C}^{n \times n} \mid \operatorname{rk}(A)=\right.$ $\left.\operatorname{rk}\left(A^{2}\right)\right\}$.

The first author is the corresponding author. 
Let $A \in \mathbb{C}^{n \times n}$. A matrix $X \in \mathbb{C}^{n \times n}$ such that $X A^{k+1}=A^{k}, X A X=X$ and $A X=X A$ is called the Drazin inverse of $A$ and denoted by $A^{D}$. The Drazin inverse of a square matrix always exists and it is unique. Such integer $k$ is called the Drazin index of $A$, denoted by $\operatorname{ind}(A)$. If $\operatorname{ind}(A) \leq 1$, then the Drazin inverse of $A$ is called the group inverse and denoted by $A^{\#}$.

The DMP-inverse for a complex matrix was introduced by Malik and Thome [9]. Let $A \in \mathbb{C}^{n \times n}$ with ind $(A)=k$. A matrix $X \in \mathbb{C}^{n \times n}$ is called a $D M P$-inverse of $A$, if it satisfies $X A X=X, X A=A^{D} A$ and $A^{k} X=A^{k} A^{\dagger}$. It is unique and denoted by $A^{D, \dagger}$. Malik and Thome gave several characterizations of the DMP-inverse by using the decomposition of Hartwig and Spindelböck [8].

The notion of the core-EP inverse for a complex matrix was introduced by Manjunatha Prasad and Mohana [10]. A matrix $X \in \mathbb{C}^{n \times n}$ is a core-EP inverse of $A \in \mathbb{C}^{n \times n}$ if $X$ is an outer inverse of $A$ satisfying $\mathcal{R}(X)=\mathcal{R}\left(X^{*}\right)=\mathcal{R}\left(A^{k}\right)$, where $k$ is the index of $A$. The core-EP inverse is unique and denoted by $A$.

In addition, $\mathbf{1}_{n}$ and $\mathbf{0}_{n}$ will denote the $n \times 1$ column vectors all of whose components are 1 and 0 , respectively. $0_{m \times n}$ (abbr. 0 ) denotes the zero matrix of size $m \times n$. If $\delta$ is a subspace of $\mathbb{C}^{n}$, then $P_{\delta}$ stands for the orthogonal projector onto the subspace 8. A matrix $A \in \mathbb{C}^{n \times n}$ is called an $E P$ matrix if $\mathcal{R}(A)=\mathcal{R}\left(A^{*}\right), A$ is called Hermitian if $A^{*}=A$ and $A$ is unitary if $A A^{*}=I_{n}$, where $I_{n}$ denote the identity matrix of size $n$. Let $\mathbb{N}$ denote the set of positive integers.

\section{PReliminaries}

A related decomposition of the matrix decomposition of Hartwig and Spindelböck [8] was given in [2, Theorem 2.1] by Benítez, in [3] it can be found a simpler proof of this decomposition. Let us start this section with the concept of principal angles.

Definition 1 ([12]). Let $\delta_{1}$ and $\delta_{2}$ be two nontrivial subspaces of $\mathbb{C}^{n}$. We define the principal angles $\theta_{1}, \ldots, \theta_{r} \in[0, \pi / 2]$ between $\delta_{1}$ and $\delta_{2}$ by

$$
\cos \theta_{i}=\sigma_{i}\left(P_{\delta_{1}} P_{\delta_{2}}\right),
$$

for $i=1, \ldots, r$, where $r=\min \left\{\operatorname{dim} \S_{1}, \operatorname{dim} \S_{2}\right\}$. The real numbers $\sigma_{i}\left(P_{\delta_{1}} P_{\delta_{2}}\right) \geq 0$ are the singular values of $P_{\delta_{1}} P_{\delta_{2}}$.

The following theorem can be found in [2, Theorem 2.1].

Theorem 1. Let $A \in \mathbb{C}^{n \times n}, r=\operatorname{rk}(A)$, and let $\theta_{1}, \ldots, \theta_{p}$ be the principal angles between $\mathcal{R}(A)$ and $\mathcal{R}\left(A^{*}\right)$ belonging to $] 0, \pi / 2[$. Denote by $x$ and $y$ the multiplicities of the angles 0 and $\pi / 2$ as a canonical angle between $\mathcal{R}(A)$ and $\mathcal{R}\left(A^{*}\right)$, respectively. There exists a unitary matrix $U \in \mathbb{C}^{n \times n}$ such that

$$
A=U\left[\begin{array}{cc}
M C & M S \\
0 & 0
\end{array}\right] U^{*}
$$


where $M \in \mathbb{C}^{r \times r}$ is nonsingular,

$$
\begin{gathered}
C=\operatorname{diag}\left(\mathbf{0}_{y}, \cos \theta_{1}, \ldots, \cos \theta_{p}, \mathbf{1}_{x}\right), \\
S=\left[\begin{array}{cc}
\operatorname{diag}\left(\mathbf{1}_{y}, \sin \theta_{1}, \ldots, \sin \theta_{p}\right) & 0_{p+y, n-(r+p+y)} \\
0_{x, p+y} & 0_{x, n-(r+p+y)}
\end{array}\right],
\end{gathered}
$$

and $r=y+p+x$. Furthermore, $x$ and $y+n-r$ are the multiplicities of the singular values 1 and 0 in $P_{\mathcal{R}(A)} P_{\mathcal{R}\left(A^{*}\right)}$, respectively.

In this decomposition, one has $C^{2}+S S^{*}=I_{r}$. Recall that $A^{\dagger}$ always exists. We have that $A^{\#}$ exists if and only if $C$ is nonsingular in view of [2, Theorem 3.7]. The following equalities hold

$$
A^{\dagger}=U\left[\begin{array}{cc}
C M^{-1} & 0 \\
S^{*} M^{-1} & 0
\end{array}\right] U^{*}, \quad A^{\#}=U\left[\begin{array}{cc}
C^{-1} M^{-1} & C^{-1} M^{-1} C^{-1} S \\
0 & 0
\end{array}\right] U^{*} .
$$

By [3, Theorem 2], we have that

$$
A^{D}=U\left[\begin{array}{cc}
(M C)^{D} & {\left[(M C)^{D}\right]^{2} M S} \\
0 & 0
\end{array}\right] U^{*} .
$$

We also have

$$
\begin{gathered}
A A^{\dagger}=U\left[\begin{array}{cc}
I_{r} & 0 \\
0 & 0
\end{array}\right] U^{*}, \\
A^{\boxplus}=A^{\#} A A^{\dagger}=U\left[\begin{array}{cc}
C^{-1} M^{-1} & 0 \\
0 & 0
\end{array}\right] U^{*} .
\end{gathered}
$$

Lemma 1 ([13, Theorem 3.1]). Let $A \in \mathbb{C}^{n \times n}$. Then $A$ is core invertible if and only if there exists $X \in \mathbb{C}^{n \times n}$ such that $(A X)^{*}=A X, X A^{2}=A$ and $A X^{2}=X$. In this situation, we have $A^{\boxplus}=X$.

Lemma 2. Let $A \in \mathbb{C}^{n \times n}$. If there exists $X \in \mathbb{C}^{n \times n}$ such that $A X^{k+1}=X^{k}$ and $X A^{k+1}=A^{k}$ for some $k \in \mathbb{N}$, then for $m \in \mathbb{N}$ we have
(1) $A^{k}=X^{m} A^{k+m}$;
(2) $X^{k}=A^{m} X^{k+m}$;
(3) $A^{k} X^{k}=A^{k+m} X^{k+m}$.
(4) $X^{k} A^{k}=X^{k+m} A^{k+m}$;
(5) $A^{k}=A^{m} X^{m} A^{k}$;
(6) $X^{k}=X^{m} A^{m} X^{k}$.

Proof. (1). For $m=1$, it is clear by the hypotheses. If the formula is true for $m \in \mathbb{N}$, then $X^{m+1} A^{k+m+1}=X X^{m} A^{k+m} A=X A^{k} A=X A^{k+1}=A^{k}$.

(3). It is easy to check that $A^{k} X^{k}=A^{k+1} X^{k+1}$ by $A X^{k+1}=X^{k}$. It is not difficult to check the equality $A^{k} X^{k}=A^{k+m} X^{k+m}$ by induction.

(5). From (1) we have $A^{k}=X^{k} A^{2 k}$. Thus by $A X^{k+1}=X^{k}$, we have $A^{k}=$ $X^{k} A^{2 k}=A X^{k+1} A^{2 k}=A X^{k} X A^{2 k}=A\left(A X^{k+1}\right) X A^{2 k}=A^{2} X^{k+2} A^{2 k}=$ $A^{2} X^{2} X^{k} A^{2 k}=\cdots=A^{m} X^{m} X^{k} A^{2 k}=A^{m} X^{m} A^{k}$. 
The proofs of (2), (4), and (6) are similar to the proofs of (1), (3), (5), respectively.

Lemma 3. Let $A \in \mathbb{C}^{n \times n}$. If there exists $X \in \mathbb{C}^{n \times n}$ such that $A X^{k+1}=X^{k}$ and $X A^{k+1}=A^{k}$ for some $k \in \mathbb{N}$, then $A^{D}=X^{k+1} A^{k}$.

Proof. Since $A$ is Drazin invertible. We will check that $A^{D}=X^{k+1} A^{k}$. Have in mind, $A X^{k+1}=X^{k}$ and $X A^{k+1}=A^{k}$, thus

$$
A\left(X^{k+1} A^{k}\right)=X^{k} A^{k}=X^{k}\left(X A^{k+1}\right)=X^{k+1} A^{k} A .
$$

That is, $X^{k+1} A^{k}$ and $A$ commute. Then by (1) and (4) in Lemma 2, we have that

$$
\begin{aligned}
\left(X^{k+1} A^{k}\right) A\left(X^{k+1} A^{k}\right) & =X^{k+1} A^{k+1} X^{k+1} A^{k}=X^{k} A^{k}\left(X^{k+1} A^{k}\right) \\
& =X^{k} X^{k+1} A^{k} A^{k}=X^{k+1} X^{k} A^{2 k}=X^{k+1} A^{k} .
\end{aligned}
$$

From (1) in Lemma 2, we have that

$$
\left(X^{k+1} A^{k}\right) A^{k+1}=X\left(X^{k} A^{2 k}\right) A=X A^{k+1}=A^{k} .
$$

Thus we have $A^{D}=X^{k+1} A^{k}$ by the definition of the Drazin inverse and in view of (2.5), (2.6), and (2.7).

Remark 1. From the proofs of Lemma 2 and Lemma 3, it is obvious that Lemma 2 and Lemma 3 are valid for rings. Moreover, we can get that for an element $a \in R, a$ is Drazin invertible if and only if there exist $x \in R$ and $k \in \mathbb{N}$ such that $a x^{k+1}=x^{k}$ and $x a^{k+1}=a^{k}$, where $R$ is a ring.

The following lemma is similar to [9, Theorem 2.5].

Lemma 4. Let $A \in \mathbb{C}^{n \times n}$ be the form (2.1). Then

$$
A^{D, \dagger}=U\left[\begin{array}{cc}
(M C)^{D} & 0 \\
0 & 0
\end{array}\right] U^{*} .
$$

Proof. By (2.2), (2.3) and the definition of DMP-inverse we have

$$
\begin{aligned}
A^{D, \dagger} & =A^{D} A A^{\dagger} \\
& =U\left[\begin{array}{cc}
(M C)^{D} & {\left[(M C)^{D}\right]^{2} M S} \\
0 & 0
\end{array}\right]\left[\begin{array}{cc}
I_{r} & 0 \\
0 & 0
\end{array}\right] U^{*}=U\left[\begin{array}{cc}
(M C)^{D} & 0 \\
0 & 0
\end{array}\right] U^{*} .
\end{aligned}
$$

Lemma 5 ([11, Corollary 3.3]). Let $A \in \mathbb{C}^{n \times n}$ be a matrix of index $k$. Then $A A^{\oplus}=A^{k}\left(A^{k}\right)^{\dagger}$. 


\section{3. $\langle i, m\rangle$-CORE INVERSE}

Let us start this section by introducing the definition of the $\langle i, m\rangle$-core inverse.

Definition 2. Let $A \in \mathbb{C}^{n \times n}$ and $m, i \in \mathbb{N}$. A matrix $X \in \mathbb{C}^{n \times n}$ is called an $\langle i, m\rangle$ core inverse of $A$, if it satisfies

$$
X=A^{D} A X \quad \text { and } \quad A^{m} X=A^{i}\left(A^{i}\right)^{\dagger} .
$$

It will be proved that if $X$ exists, then it is unique and denoted by $A_{i, m}^{\oplus}$.

Theorem 2. Let $A \in \mathbb{C}^{n \times n}$. If exists $X \in \mathbb{C}^{n \times n}$ such that (3.1) holds, then $X$ is unique.

Proof. Assume that $X$ satisfies the system in (3.1), that is $X=A^{D} A X$ and $A^{m} X=$ $A^{i}\left(A^{i}\right)^{\dagger}$. Thus $X=A^{D} A X=\left(A^{D}\right)^{m} A^{m} X=\left(A^{D}\right)^{m} A^{i}\left(A^{i}\right)^{\dagger}$. Therefore, $X$ is unique by the uniqueness of $A^{D}$ and $A^{i}\left(A^{i}\right)^{\dagger}$.

Theorem 3. The system in (3.1) is consistent if and only if $i \geq \operatorname{ind}(A)$. In this case, the solution of (3.1) is $X=\left(A^{D}\right)^{m} A^{i}\left(A^{i}\right)^{\dagger}$.

Proof. Assume that $i \geq \operatorname{ind}(A)$. Let $X=\left(A^{D}\right)^{m} A^{i}\left(A^{i}\right)^{\dagger}$. We have

$$
\begin{aligned}
A^{D} A X & =A^{D} A\left(A^{D}\right)^{m} A^{i}\left(A^{i}\right)^{\dagger}=\left(A^{D}\right)^{m} A^{D} A A^{i}\left(A^{i}\right)^{\dagger}=\left(A^{D}\right)^{m} A^{i}\left(A^{i}\right)^{\dagger}=X \\
A^{m} X & =A^{m}\left(A^{D}\right)^{m} A^{i}\left(A^{i}\right)^{\dagger}=A^{D} A A^{i}\left(A^{i}\right)^{\dagger}=A^{i}\left(A^{i}\right)^{\dagger} .
\end{aligned}
$$

Thus, the system in (3.1) is consistent and the solution of (3.1) is $X=\left(A^{D}\right)^{m} A^{i}\left(A^{i}\right)^{\dagger}$.

If the system in (3.1) is consistent, then exists $X_{0}$ such that $X_{0}=A^{D} A X_{0}$ and $A^{m} X_{0}=A^{i}\left(A^{i}\right)^{\dagger}$. Then $X_{0}=A^{D} A X_{0}=\left(A^{D}\right)^{m} A^{m} X_{0}=\left(A^{D}\right)^{m} A^{i}\left(A^{i}\right)^{\dagger}$ and $A^{i}\left(A^{i}\right)^{\dagger}=A^{m} X_{0}=A^{m}\left(A^{D}\right)^{m} A^{i}\left(A^{i}\right)^{\dagger}=A A^{D} A^{i}\left(A^{i}\right)^{\dagger}$. Hence $A^{i}=A^{i}\left(A^{i}\right)^{\dagger} A^{i}=$ $A A^{D} A^{i}\left(A^{i}\right)^{\dagger} A^{i}=A A^{D} A^{i}$, that is $i \geq \operatorname{ind}(A)$.

Example 1. We will give an example that shows if $i<\operatorname{ind}(A)$, then the system in (3.1) is not consistent. Let $A=\left[\begin{array}{ll}0 & 1 \\ 0 & 0\end{array}\right]$. It is easy to get $\operatorname{ind}(A)=2$ and $A^{D}=0$. Let $i=1$ and suppose that $X$ is the solution of system in (3.1), then $X=A^{D} A X=0$, which gives $A A^{\dagger}=A^{m} X=0$, thus $A=A A^{\dagger} A=0$, this is a contradiction.

Remark 2. If $i \geq \operatorname{ind}(A)$, then $A_{i, m+1}^{\oplus}=A^{D} A_{i, m}^{\oplus}$.

Remark 3. The $\langle i, m\rangle$-core inverse is a generalization of the core inverse and the core-EP inverse. More precisely, we have the following statements:

(1) If $m=i=\operatorname{ind}(A)=1$, then the $\langle 1,1\rangle$-core inverse coincides with the core inverse;

(2) If $m=1$ and $i=\operatorname{ind}(A)$, then the $\langle i, 1\rangle$-core inverse coincides with the coreEP inverse. 
For the convenience of the readers, in the following, we give some notes of (1) and (2) in Remark 3.

(1) If $m=i=\operatorname{ind}(A)=1$, then $A$ is group invertible and $A^{D}=A^{\#}$ and (3.1) is equivalent to $X=A^{\#} A X$ and $A X=A A^{\dagger}$. Thus $X=A^{\#} A X=A^{\#} A A^{\dagger}$, $(A X)^{*}=\left(A A^{\dagger}\right)^{*}=A A^{\dagger}=A X, A X^{2}=A A^{\#} A A^{\dagger} A^{\#} A A^{\dagger}=A A^{\#} A A^{\dagger} A A^{\#} A^{\dagger}=$ $A A^{\#} A^{\dagger}=X$ and $X A^{2}=A^{\#} A A^{\dagger} A^{2}=A^{\#} A^{2}=A$. Hence, $\langle 1,1\rangle$-core inverse coincides with the core inverse by Lemma 1 . Note that if $A$ is group invertible, then we have that $X$ is the core inverse of $A$ if and only if $X=A^{\#} A X$ and $A X=A A^{\dagger}$.

(2) If $m=1$ and $i=\operatorname{ind}(A)$, then by Theorem 3.3, $A_{i, 1}^{\oplus}$ exists and $A_{i, 1}^{\oplus}=$ $A^{D} A^{i}\left(A^{i}\right)^{\dagger}$. Let us denote $X=A_{i, 1}^{\oplus}=A^{D} A^{i}\left(A^{i}\right)^{\dagger}$. Observe that $A X=$ $A^{i}\left(A^{i}\right)^{\dagger}$ is Hermitian. Now,

$$
X A X=A^{D} A^{i}\left(A^{i}\right)^{\dagger} A^{i}\left(A^{i}\right)^{\dagger}=A^{D} A^{i}\left(A^{i}\right)^{\dagger}=X,
$$

that is $X$ is an outer inverse of $A$. From

$$
A^{i}=A^{D} A^{i+1}=A^{D} A^{i}\left(A^{i}\right)^{\dagger} A^{i} A=X A^{i+1}
$$

we get $\mathcal{R}\left(A^{i}\right) \subseteq \mathcal{R}(X)$. Also,

$$
\begin{aligned}
A X^{2} & =(A X) X=A^{i}\left(A^{i}\right)^{\dagger} A^{D} A^{i}\left(A^{i}\right)^{\dagger} \\
& =A^{i}\left(A^{i}\right)^{\dagger} A^{i} A^{D}\left(A^{i}\right)^{\dagger}=A^{D} A^{i}\left(A^{i}\right)^{\dagger}=X,
\end{aligned}
$$

which implies $X=(A X)^{*} X \in \mathcal{R}\left(X^{*}\right)$, therefore, $\mathcal{R}(X) \subseteq \mathcal{R}\left(X^{*}\right)$. Finally, $X^{*}=\left[A^{D} A^{i}\left(A^{i}\right)^{\dagger}\right]^{*}=A^{i}\left(A^{i}\right)^{\dagger}\left(A^{D}\right)^{*}$ implies $\mathcal{R}\left(X^{*}\right) \subseteq \mathcal{R}\left(A^{i}\right)$. Hence $\mathcal{R}(X)=\mathcal{R}\left(X^{*}\right)=\mathcal{R}\left(A^{i}\right)$. Therefore, the $\langle i, 1\rangle$-core inverse coincides with the core-EP inverse by the definition of the core-EP inverse.

From the above statement, we have the following theorem.

Theorem 4. Let $A \in \mathbb{C}^{n \times n}$ with $i=\operatorname{ind}(A)$. Then $X$ is the core-EP inverse of $A$ if and only if $X=A^{D} A X$ and $A X=A^{i}\left(A^{i}\right)^{\dagger}$.

Corollary 1. Let $A \in \mathbb{C}^{n \times n}$ with $1=\operatorname{ind}(A)$. Then $X$ is the core inverse of $A$ if and only if $X=A^{\#} A X$ and $A X=A A^{\dagger}$.

For any $A \in \mathbb{C}^{n \times n}$, either $A^{l}=0$ for some $l \in \mathbb{N}$, or $A^{l} \neq 0$ for all positive integers. Moreover, if $\operatorname{ind}(A)=k$, then $G_{k} B_{k}$ is nonsingular (see [5-7]), where $A=B_{1} G_{1}$ is a full rank factorization of $A$ and $G_{l} B_{l}=B_{l+1} G_{l+1}$ is a full rank factorization of $G_{l} B_{l}, l=1, \ldots, k-1$. When $A^{k} \neq 0$, then it can be written as

$$
A^{k}=\prod_{l=1}^{k} B_{l} \prod_{l=1}^{k} G_{k+1-l} .
$$


We have the following results, (see [5, Theorem 4] or [4, Theorem 7.8.2]):

$$
\operatorname{ind}(A)= \begin{cases}k & \text { when } G_{k} B_{k} \text { is nonsingular, } \\ k+1 & \text { when } G_{k} B_{k}=0\end{cases}
$$

and

$$
A^{D}= \begin{cases}\prod_{l=1}^{k} B_{l}\left(G_{k} B_{k}\right)^{-k-1} \prod_{l=1}^{k} G_{k+1-l} & \text { when } G_{k} B_{k} \text { is nonsingular, } \\ 0 & \text { when } G_{k} B_{k}=0 .\end{cases}
$$

In the sequel, we always assume that $A^{k} \neq 0$.

It is well-known that if $A=E F$ is a full rank factorization of $A$, where $r=\operatorname{rk}(A)$, $E \in \mathbb{C}^{n \times r}$ and $F \in \mathbb{C}^{r \times n}$, then (see [4, Theorem 1.3.2])

$$
A^{\dagger}=F^{*}\left(F F^{*}\right)^{-1}\left(E^{*} E\right)^{-1} E^{*} .
$$

Remark 4. The notations and results in above paragraph will be used many times in the sequel.

We will investigate the $\langle i, m\rangle$-core inverse of a matrix $A \in \mathbb{C}^{n \times n}$ by using Remark 4.

Theorem 5. Let $A \in \mathbb{C}^{n \times n}$ with $\operatorname{ind}(A)=k$. If $i \geq k$, then $A_{i, m}^{\oplus}=A_{k, m}^{\oplus}$.

Proof. Since ind $(A)=k$, we have $\mathcal{R}\left(A^{k}\right)=\mathcal{R}\left(A^{i}\right)$ for any $i \geq k$, and therefore, $A^{k}\left(A^{k}\right)^{\dagger}=A^{i}\left(A^{i}\right)^{\dagger}$. Now, the conclusion follows from Theorem 3 .

Remark 5. The proof of Theorem 5 also can be proved as follows. Since the proof in this remark will be used several times in the sequel, we write this proof here.

Proof. If $A$ is nilpotent, then $A^{D}=0$, hence by Theorem 3, one has $A_{i, m}^{\oplus}=$ $A_{k, m}^{\oplus}=0$. Therefore, we can assume that $A^{k} \neq 0$. By equality (3.2), we have

$$
A^{k}=\prod_{l=1}^{k} B_{l} \prod_{l=1}^{k} G_{k+1-l}
$$

where $A=B_{1} G_{1}$ is a full rank factorization of $A$ and $G_{l} B_{l}=B_{l+1} G_{l+1}$ is a full rank factorization of $G_{l} B_{l}, l=1, \ldots, k-1$. Let $M=\prod_{l=1}^{k} B_{l}, N=\prod_{l=1}^{k} G_{k+1-l}$ and $L=G_{k} B_{k}$. Now, we will show that

$$
A^{i}=\prod_{l=1}^{k} B_{l}\left(G_{k} B_{k}\right)^{i-k} \prod_{l=1}^{k} G_{k+1-l}=M L^{i-k} N .
$$


In fact,

$$
\begin{aligned}
A^{i} & =\prod_{l=1}^{i} B_{l} \prod_{l=1}^{i} G_{k+1-l} \\
& =B_{1} \cdots B_{i} G_{i} \cdots G_{1} \\
& =B_{1} \cdots B_{i-1}\left(B_{i} G_{i}\right) G_{i-1} \cdots G_{1} \\
& =B_{1} \cdots B_{i-1}\left(G_{i-1} B_{i-1}\right) G_{i-1} \cdots G_{1} \\
& =B_{1} \cdots B_{i-2}\left(G_{i-2} B_{i-2}\right)^{2} G_{i-2} \cdots G_{1} \\
& =\cdots \\
& =B_{1} \cdots B_{k}\left(G_{k} B_{k}\right)^{i-k} G_{k} \cdots G_{1}=M L^{i-k} N .
\end{aligned}
$$

If we let $M_{1}=M L^{i-k}$, then $A^{i}=M L^{i-k} N=M_{1} N$ is a full rank factorization of $A^{i}$ (see [7, p.183]). Thus

$$
\left(A^{i}\right)^{\dagger}=N^{*}\left(N N^{*}\right)^{-1}\left(M_{1}^{*} M_{1}\right)^{-1} M_{1}^{*} .
$$

Note that $N M=\prod_{l=1}^{k} G_{k+1-l} \prod_{l=1}^{k} B_{l}=L^{k}$. By Theorem 3, (3.3) and (3.7) we have

$$
\begin{aligned}
A_{i, 1}^{\oplus} & =A^{D} A^{i}\left(A^{i}\right)^{\dagger} \\
& =M L^{-k-1} N M L^{i-k} N\left(A^{i}\right)^{\dagger} \\
& =M L^{-k-1} N M L^{i-k} N N^{*}\left(N N^{*}\right)^{-1}\left(M_{1}^{*} M_{1}\right)^{-1} M_{1}^{*} \\
& =M L^{i-k-1} N N^{*}\left(N N^{*}\right)^{-1}\left(M_{1}^{*} M_{1}\right)^{-1} M_{1}^{*} \\
& =M L^{i-k-1}\left(M_{1}^{*} M_{1}\right)^{-1} M_{1}^{*} \\
& =M L^{i-k-1}\left[\left(L^{i-k}\right)^{*} M^{*} M L^{i-k}\right]^{-1}\left(L^{i-k}\right)^{*} M^{*} \\
& =M L^{i-k-1} L^{k-i}\left(M^{*} M\right)^{-1}\left[\left(L^{i-k}\right)^{*}\right]^{-1}\left(L^{i-k}\right)^{*} M^{*} \\
& =M L^{-1}\left(M^{*} M\right)^{-1} M^{*} .
\end{aligned}
$$

The last expression does not depend on $i$, then $A_{i, 1}^{\oplus}=A_{k, 1}^{\oplus}$. Thus, by Remark 2, we have $A_{i, m}^{\oplus}=A^{D} A_{i, m-1}^{\oplus}=A^{D}\left(A^{D} A_{i, m-2}^{\oplus}\right)=\left(A^{D}\right)^{2} A_{i, m-2}^{\oplus,}=\cdots=\left(A^{D}\right)^{m-1} A_{i, 1}^{\oplus}=$ $\left(A^{D}\right)^{m-1} A_{k, 1}^{\oplus}=A_{k, m}^{\oplus}$.

Remark 6. By Theorem 5, it is enough to investigate the $i=\operatorname{ind}(A)=k$ case, when we discuss the $\langle i, m\rangle$-core inverse of a matrix $A \in \mathbb{C}^{n \times n}$. That is, the Theorem 5 is a key theorem.

Theorem 6. Let $A \in \mathbb{C}^{n \times n}$ with $\operatorname{ind}(A)=k$ and $k, m \in \mathbb{N}$. If $A=B_{1} G_{1}$ is a full rank factorization of $A$ and $G_{l} B_{l}=B_{l+1} G_{l+1}$ is a full rank factorization of $G_{l} B_{l}$, 
$l=1, \ldots, k-1$, then $A_{k, m}^{\oplus}=M L^{-m} M^{\dagger}$, where $M=\prod_{l=1}^{k} B_{l}, N=\prod_{l=1}^{k} G_{k+1-l}$ and $L=G_{k} B_{k}$.

Proof. By the proof of Remark 5, we have $A_{k, 1}^{\oplus}=M L^{-1}\left(M^{*} M\right)^{-1} M^{*}$ and $N M=L^{k}$. Now, we will prove $\left(A^{D}\right)^{s} A_{k, 1}^{\oplus}=M L^{-s-1}\left(M^{*} M\right)^{-1} M^{*}$ for any $s \in \mathbb{N}$. By (3.3) we have $A^{D}=\prod_{l=1}^{k} B_{l}\left(G_{k} B_{k}\right)^{-k-1} \prod_{l=1}^{k} G_{k+1-l}=M L^{-k-1} N$. When $s=1$, we have

$$
\begin{aligned}
A^{D} A_{k, 1}^{\oplus} & =M L^{-k-1} N M L^{-1}\left(M^{*} M\right)^{-1} M^{*}=M L^{-k-1}(N M) L^{-1}\left(M^{*} M\right)^{-1} M^{*} \\
& =M L^{-k-1} L^{k} L^{-1}\left(M^{*} M\right)^{-1} M^{*}=M L^{-2}\left(M^{*} M\right)^{-1} M^{*} .
\end{aligned}
$$

Assume that $\left(A^{D}\right)^{s-1} A_{k, 1}^{\oplus}=M L^{-s}\left(M^{*} M\right)^{-1} M^{*}$. Then

$$
\begin{aligned}
\left(A^{D}\right)^{s} A_{k, 1}^{\oplus} & =A^{D}\left(A^{D}\right)^{s-1} A_{k, 1}^{\oplus}=A^{D} M L^{-s}\left(M^{*} M\right)^{-1} M^{*} \\
& =M L^{-k-1} N M L^{-s}\left(M^{*} M\right)^{-1} M^{*} \\
& =M L^{-k-1} L^{k} L^{-s}\left(M^{*} M\right)^{-1} M^{*} \\
& =M L^{-s-1}\left(M^{*} M\right)^{-1} M^{*} .
\end{aligned}
$$

Thus by Remark 2, we have

$$
A_{k, m}^{\oplus}=\left(A^{D}\right)^{m-1} A_{k, 1}^{\oplus}=M L^{-m}\left(M^{*} M\right)^{-1} M^{*}=M L^{-m} M^{\dagger} .
$$

In the following theorem, we will give a canonical form for the $\langle k, m\rangle$-core inverse of a matrix $A \in \mathbb{C}^{n \times n}$ by using the matrix decomposition in Theorem 1 . We will also use the following simple fact: Let $X \in \mathbb{C}^{n \times m}$ and $\mathbf{b} \in \mathbb{C}^{n}$. If $\mathbf{y} \in \mathbb{C}^{m}$ satisfies $X^{*} X \mathbf{y}=X^{*} \mathbf{b}$, then $X X^{\dagger} \mathbf{b}=X \mathbf{y}$.

Theorem 7. Let $A \in \mathbb{C}^{n \times n}$ have the form (2.1) with $\operatorname{ind}(A)=k$ and $m \in \mathbb{N}$. Then

$$
A_{k, m}^{\oplus}=U\left[\begin{array}{cc}
(M C)_{k-1, m}^{\oplus} & 0 \\
0 & 0
\end{array}\right] U^{*} .
$$

Proof. Let $r$ be the rank of $A$. By Theorem 3 we have

$$
A_{k, m}^{\oplus}=\left(A^{D}\right)^{m} A^{k}\left(A^{k}\right)^{\dagger} .
$$

Since $A$ has the form given in Theorem 1 we have

$$
A^{k}=U\left[\begin{array}{cc}
(M C)^{k} & (M C)^{k-1} M S \\
0 & 0
\end{array}\right] U^{*} .
$$

Let $\mathbf{b} \in \mathbb{C}^{n}$ be arbitrary and let us decompose $\mathbf{b}=U\left[\begin{array}{l}\mathbf{b}_{1} \\ \mathbf{b}_{2}\end{array}\right]$, where $\mathbf{b}_{1} \in \mathbb{C}^{r}$. Let $\mathbf{x}_{0} \in \mathbb{C}^{n}$ satisfy $\left(A^{k}\right)^{*} A^{k} \mathbf{x}_{0}=\left(A^{k}\right)^{*} \mathbf{b}$ [this $\mathbf{x}_{0}$ always exists because the normal 
equations always have a solution]. We can decompose $\mathbf{x}_{0}$ by writing $\mathbf{x}_{0}=U\left[\begin{array}{l}\mathbf{x}_{1} \\ \mathbf{x}_{2}\end{array}\right]$, where $\mathbf{x}_{1} \in \mathbb{C}^{r}$. Let us denote $N=(M C)^{k-1} M$. Using (3.11),

$$
U\left[\begin{array}{cc}
C N^{*} & 0 \\
S^{*} N^{*} & 0
\end{array}\right]\left[\begin{array}{cc}
N C & N S \\
0 & 0
\end{array}\right]\left[\begin{array}{l}
\mathbf{x}_{1} \\
\mathbf{x}_{2}
\end{array}\right]=U\left[\begin{array}{cc}
C N^{*} & 0 \\
S^{*} N^{*} & 0
\end{array}\right]\left[\begin{array}{l}
\mathbf{b}_{1} \\
\mathbf{b}_{2}
\end{array}\right] .
$$

Therefore,

$$
C N^{*} N\left(C \mathbf{x}_{1}+S \mathbf{x}_{2}\right)=C N^{*} \mathbf{b}_{1} \text { and } S^{*} N^{*} N\left(C \mathbf{x}_{1}+S \mathbf{x}_{2}\right)=S^{*} N^{*} \mathbf{b}_{1} .
$$

Premultiplying the first equality by $C$ and the second equality by $S$ and after, adding them, we get $N^{*} N\left(C \mathbf{x}_{1}+S \mathbf{x}_{2}\right)=N^{*} \mathbf{b}_{1}$, and hence, $N\left(C \mathbf{x}_{1}+S \mathbf{x}_{2}\right)=N N^{\dagger} \mathbf{b}_{1}$. Now,

$$
\begin{aligned}
A^{k}\left(A^{k}\right)^{\dagger} \mathbf{b} & =A^{k} \mathbf{x}_{0}=U\left[\begin{array}{cc}
N C & N S \\
0 & 0
\end{array}\right]\left[\begin{array}{l}
\mathbf{x}_{1} \\
\mathbf{x}_{2}
\end{array}\right] \\
& =U\left[\begin{array}{c}
N C \mathbf{x}_{1}+N S \mathbf{x}_{2} \\
\mathbf{0}
\end{array}\right]=U\left[\begin{array}{c}
N N^{\dagger} \mathbf{b}_{1} \\
\mathbf{0}
\end{array}\right]=U\left[\begin{array}{cc}
N N^{\dagger} & 0 \\
0 & 0
\end{array}\right] U^{*} \mathbf{b} .
\end{aligned}
$$

Since $\mathbf{b}$ is arbitrary,

$$
A^{k}\left(A^{k}\right)^{\dagger}=U\left[\begin{array}{cc}
N N^{\dagger} & 0 \\
0 & 0
\end{array}\right] U^{*} .
$$

Now we will prove $N N^{\dagger}=(M C)^{k-1}\left[(M C)^{k-1}\right]^{\dagger}$. Recall that we have $N=$ $(M C)^{k-1} M$, and so, $\mathcal{R}(N) \subseteq \mathcal{R}\left((M C)^{k-1}\right)$. Since $M$ is nonsingular, $\operatorname{rk}(N)=$ $\operatorname{rk}\left((M C)^{k-1}\right)$, and thus, $\mathcal{R}(N)=\mathcal{R}\left((M C)^{k-1}\right)$. Since $(M C)^{k-1}\left[(M C)^{k-1}\right]^{\dagger}$ and $N N^{\dagger}$ are the orthogonal projectors onto $\mathcal{R}\left((M C)^{k-1}\right)$ and $\mathcal{R}(N)$, respectively, we get $N N^{\dagger}=(M C)^{k-1}\left[(M C)^{k-1}\right]^{\dagger}$.

By (2.2) we have

$$
A^{D}=U\left[\begin{array}{cc}
(M C)^{D} & {\left[(M C)^{D}\right]^{2} M S} \\
0 & 0
\end{array}\right] U^{*} .
$$

Thus, we have

$$
\left(A^{D}\right)^{m}=U\left[\begin{array}{cc}
{\left[(M C)^{D}\right]^{m}} & {\left[(M C)^{D}\right]^{m+1} M S} \\
0 & 0
\end{array}\right] U^{*} .
$$

Since ind $(A)=k$, we have $A^{D} A^{k+1}=A^{k}$. By using the above representations of $A^{D}$ and $A^{k}$ given in (3.11) and (3.12), respectively,

$$
\begin{aligned}
& {\left[\begin{array}{cc}
(M C)^{D} & {\left[(M C)^{D}\right]^{2} M S} \\
0 & 0
\end{array}\right]\left[\begin{array}{cc}
(M C)^{k+1} & (M C)^{k} M S \\
0 & 0
\end{array}\right] } \\
&=\left[\begin{array}{cc}
(M C)^{k} & (M C)^{k-1} M S \\
0 & 0
\end{array}\right] .
\end{aligned}
$$

Therefore,

$$
(M C)^{D}(M C)^{k} M[C \mid S]=(M C)^{k-1} M[C \mid S] .
$$


Have in mind that we have $C^{2}+S S^{*}=I_{r}$. Thus, postmultiplying (3.13) by $\left[\begin{array}{c}C \\ S^{*}\end{array}\right]$ gives us $(M C)^{D}(M C)^{k} M=(M C)^{k-1} M$ and from the nonsningularity of $M$ we obtain $(M C)^{D}(M C)^{k}=(M C)^{k-1}$, and so, $\operatorname{ind}(M C) \leq k-1$. Therefore we have

$$
\begin{aligned}
A_{k, m}^{\oplus} & =\left(A^{D}\right)^{m} A^{k}\left(A^{k}\right)^{\dagger} \\
& =U\left[\begin{array}{cc}
{\left[(M C)^{D}\right]^{m}} & {\left[(M C)^{D}\right]^{m+1} M S} \\
0 & 0
\end{array}\right]\left[\begin{array}{cc}
(M C)^{k-1}\left((M C)^{k-1}\right)^{\dagger} & 0 \\
0 & 0
\end{array}\right] U^{*} \\
& =U\left[\begin{array}{cc}
{\left[(M C)^{D}\right]^{m}(M C)^{k-1}\left((M C)^{k-1}\right)^{\dagger}} & 0 \\
0 & 0
\end{array}\right] U^{*} \\
& =U\left[\begin{array}{cc}
(M C)_{k-1, m}^{\oplus} & 0 \\
0 & 0
\end{array}\right] U^{*} .
\end{aligned}
$$

Remark 7. If we use the decomposition of Hartwig and Spindelböck in [8, Corollary 6], then an expression of the $\langle k, m\rangle$-core inverse of $A$ is

$$
A_{k, m}^{\oplus}=U\left[\begin{array}{cc}
(\Sigma K)_{k-1, m}^{\oplus} & 0 \\
0 & 0
\end{array}\right] U^{*}
$$

which is similar to the expression of $A_{k, m}^{\oplus}$ in Theorem 7. Since the proof of this result can be proved as the proof of Theorem 7, we omit this proof.

Let $A \in \mathbb{C}^{n \times n}$ with ind $(A)=k$. The Jordan Canonical form of $A$ is $P^{-1} A P=J$, where $P \in \mathbb{C}^{n \times n}$ is nonsingular and $J \in \mathbb{C}^{n \times n}$ is a block diagonal matrix composed of Jordan blocks. In the following theorem, we will compute the $\langle k, m\rangle$-core inverse by using the Jordan Canonical form of $A$.

Theorem 8. Let $A \in \mathbb{C}^{n \times n}$ with $\operatorname{ind}(A)=k$, then $A_{k, m}^{\oplus}=P_{1} D^{-m} P_{1}^{\dagger}$, where $A=P\left[\begin{array}{cc}D & 0 \\ 0 & N\end{array}\right] P^{-1}$ with $D \in \mathbb{C}^{r \times r}$ is nonsingular, $N$ is nilpotent and $P=\left[P_{1} \mid P_{2}\right]$ with $P_{1} \in \mathbb{C}^{n \times r}$.

Proof. The Jordan Canonical form of $A$ is $P^{-1} A P=J$, where $P \in \mathbb{C}^{n \times n}$ is nonsingular and $J \in \mathbb{C}^{n \times n}$ is a block diagonal matrix. Rearrange the elements of $J$ such that $A=P\left[\begin{array}{ll}D & 0 \\ 0 & N\end{array}\right] P^{-1}$, where $D$ is nonsingular and $N$ is nilpotent. It is wellknown that $A^{D}=P\left[\begin{array}{cc}D^{-1} & 0 \\ 0 & 0\end{array}\right] P^{-1}$ and $A^{k}=P\left[\begin{array}{cc}D^{k} & 0 \\ 0 & 0\end{array}\right] P^{-1}$. If we let $P=\left[P_{1} \mid P_{2}\right]$ and $P^{-1}=\left[\begin{array}{l}Q_{1} \\ Q_{2}\end{array}\right]$, then

$$
\left(A^{D}\right)^{m} A^{k}=\left[P_{1} \mid P_{2}\right]\left[\begin{array}{cc}
\left(D^{-1}\right)^{m} & 0 \\
0 & 0
\end{array}\right]\left[\begin{array}{cc}
D^{k} & 0 \\
0 & 0
\end{array}\right]\left[\begin{array}{l}
Q_{1} \\
Q_{2}
\end{array}\right]=P_{1} D^{k-m} Q_{1} .
$$


Observe that $A^{k}=\left(P_{1} D^{k}\right) Q_{1}$ is a full rank factorization of $A^{k}$. Hence by (3.4) we have

$$
\begin{aligned}
\left(A^{k}\right)^{\dagger} & =\left(P_{1} D^{k} Q_{1}\right)^{\dagger} \\
& =Q_{1}^{*}\left(Q_{1} Q_{1}^{*}\right)^{-1}\left[\left(P_{1} D^{k}\right)^{*} P_{1} D^{k}\right]^{-1}\left(P_{1} D^{k}\right)^{*} \\
& =Q_{1}^{*}\left(Q_{1} Q_{1}^{*}\right)^{-1} D^{-k}\left(P_{1}^{*} P_{1}\right)^{-1}\left[\left(D^{k}\right)^{*}\right]^{-1}\left(D^{k}\right)^{*} P_{1}^{*} \\
& =Q_{1}^{*}\left(Q_{1} Q_{1}^{*}\right)^{-1} D^{-k}\left(P_{1}^{*} P_{1}\right)^{-1} P_{1}^{*} \\
& =Q_{1}^{\dagger} D^{-k} P_{1}^{\dagger} .
\end{aligned}
$$

By Theorem 3, we have $A_{k, m}^{\oplus}=\left(A^{D}\right)^{m} A^{k}\left(A^{k}\right)^{\dagger}$. Thus we have

$$
\begin{aligned}
A_{k, m}^{\oplus} & =\left(A^{D}\right)^{m} A^{k}\left(A^{k}\right)^{\dagger}=P_{1} D^{k-m} Q_{1} Q_{1}^{\dagger} D^{-k} P_{1}^{\dagger} \\
& =P_{1} D^{k-m} Q_{1} Q_{1}^{*}\left(Q_{1} Q_{1}^{*}\right)^{-1} D^{-k} P_{1}^{\dagger}=P_{1} D^{-m} D^{k} D^{-k} P_{1}^{\dagger}=P_{1} D^{-m} P_{1}^{\dagger} .
\end{aligned}
$$

Proposition 1. Let $A \in \mathbb{C}^{n \times n}$. If $i \geq \operatorname{ind}(A)$, then $A^{m} A_{i, m}^{\oplus}$ is the projector onto $\mathcal{R}\left(A^{i}\right)$ along $\mathcal{R}\left(A^{i}\right)^{\perp}$.

Proof. It is trivial.

In the following proposition, we will investigate some properties of the $\langle i, m\rangle$-core inverse.

Proposition 2. Let $A \in \mathbb{C}^{n \times n}, m, i \in \mathbb{N}$. If $i \geq \operatorname{ind}(A)$, then

(1) $A_{i, m}^{\oplus}$ is a $\{2,3\}$-inverse of $A^{m}$;

(2) $A_{i, m}^{\oplus}=\left(A^{D}\right)^{m} P_{A^{i}}$;

(3) $\left(A_{i, m}^{\oplus}\right)^{n}=\left(A^{D}\right)^{m(n-1)} A_{i, m}^{\oplus}=\left(A^{D}\right)^{m n} P_{A^{i}}$;

(4) $A^{i} A_{i, m}^{\oplus}=A_{i, m}^{\oplus} A^{i}$ if and only if $\mathcal{R}\left(A^{i}\right)^{\perp} \subseteq \mathcal{N}\left(A^{i}\right)$;

(5) $A_{i, m}^{\oplus}=A$ implies that $A$ is EP.

Proof. (1). By Theorem 3 we have $A_{i, m}^{\oplus}=\left(A^{D}\right)^{m} A^{i}\left(A^{i}\right)^{\dagger}$, thus

$$
\begin{aligned}
A_{i, m}^{\oplus} A^{m} A_{i, m}^{\oplus} & =\left(A^{D}\right)^{m} A^{i}\left(A^{i}\right)^{\dagger} A^{m}\left(A^{D}\right)^{m} A^{i}\left(A^{i}\right)^{\dagger} \\
& =\left(A^{D}\right)^{m} A^{i}\left(A^{i}\right)^{\dagger} A^{i} A^{m}\left(A^{D}\right)^{m}\left(A^{i}\right)^{\dagger} \\
& =\left(A^{D}\right)^{m} A^{i} A^{m}\left(A^{D}\right)^{m}\left(A^{i}\right)^{\dagger}=\left(A^{D}\right)^{m} A^{m}\left(A^{D}\right)^{m} A^{i}\left(A^{i}\right)^{\dagger} \\
& =A^{D} A\left(A^{D}\right)^{m} A^{i}\left(A^{i}\right)^{\dagger}=\left(A^{D}\right)^{m} A^{i}\left(A^{i}\right)^{\dagger}=A_{i, m}^{\oplus} .
\end{aligned}
$$

Thus $A_{i, m}^{\oplus}$ is a $\{2,3\}$-inverse of $A^{m}$ in view of $A^{m} A_{i, m}^{\oplus}=A^{i}\left(A^{i}\right)^{\dagger}$.

(2) is trivial. 
(3). By

$$
\begin{aligned}
\left(A_{i, m}^{\oplus}\right)^{n} & =\left(A^{D}\right)^{m} A^{i}\left(A^{i}\right)^{\dagger}\left(A^{D}\right)^{m} A^{i}\left(A^{i}\right)^{\dagger}\left(A_{i, m}^{\oplus}\right)^{n-2} \\
& =\left(A^{D}\right)^{m}\left(A^{D}\right)^{m} A^{i}\left(A^{i}\right)^{\dagger}\left(A_{i, m}^{\oplus}\right)^{n-2} \\
& =\left(A^{D}\right)^{m} A_{i, m}^{\oplus}\left(A_{i, m}^{\oplus}\right)^{n-2}=\left(A^{D}\right)^{m}\left(A_{i, m}^{\oplus}\right)^{n-1},
\end{aligned}
$$

it is easy to check (3).

(4). By $\mathcal{R}\left[\left(I_{n}-A^{i}\left(A^{i}\right)^{\dagger}\right]=\mathcal{N}\left[\left(A^{i}\right)^{\dagger}\right]\right.$, we have

$$
\begin{aligned}
A^{i} A_{i, m}^{\oplus}=A_{i, m}^{\oplus} A^{i} & \Leftrightarrow A^{i}\left(A^{D}\right)^{m} A^{i}\left(A^{i}\right)^{\dagger}=\left(A^{D}\right)^{m} A^{i}\left(A^{i}\right)^{\dagger} A^{i} \\
& \Leftrightarrow A^{i}\left(A^{D}\right)^{m} A^{i}\left(A^{i}\right)^{\dagger}=\left(A^{D}\right)^{m} A^{i} \\
& \Leftrightarrow A^{i}\left(A^{D}\right)^{m}\left(I_{n}-A^{i}\left(A^{i}\right)^{\dagger}\right)=0 \\
& \Leftrightarrow \mathcal{R}\left[I_{n}-A^{i}\left(A^{i}\right)^{\dagger}\right] \subseteq \mathcal{N}\left[A^{i}\left(A^{D}\right)^{m}\right] \\
& \Leftrightarrow \mathcal{N}\left[\left(A^{i}\right)^{\dagger}\right] \subseteq \mathcal{N}\left[\left(A^{D}\right)^{m}\right] \\
& \Leftrightarrow \mathcal{N}\left[\left(A^{i}\right)^{*}\right] \subseteq \mathcal{N}\left[\left(A^{D}\right)^{m}\right] \\
& \Leftrightarrow \mathcal{R}\left(A^{i}\right)^{\perp} \subseteq \mathcal{N}\left[\left(A^{D}\right)^{m}\right] \\
& \Leftrightarrow \mathcal{R}\left(A^{i}\right)^{\perp} \subseteq \mathcal{N}\left[A^{i}\right] .
\end{aligned}
$$

(5). Let $A$ be written in the form (2.1). We have $A_{i, m}^{\oplus}=U\left[\begin{array}{cc}(M C)_{i-1, m}^{\oplus} & 0 \\ 0 & 0\end{array}\right] U^{*}$ by Theorem 7. Thus, $A_{i, m}^{\oplus}=A$ implies $M S=0$. From the nonsingularity of $M$, we have $S=0$, which is equivalent to say that $A$ is EP in view of [2, Theorem 3.7].

\section{4. $(j, m)$-CORE INVERSE}

Let us start this section by introducing the definition of the $(j, m)$-core inverse.

Definition 3. Let $A \in \mathbb{C}^{n \times n}$ and $m, j \in \mathbb{N}$. A matrix $X \in \mathbb{C}^{n \times n}$ is called a $(j, m)$ core inverse of $A$, if it satisfies

$$
X=A^{D} A X \quad \text { and } \quad A^{m} X=A^{m}\left(A^{j}\right)^{\dagger} .
$$

Theorem 9. Let $A \in \mathbb{C}^{n \times n}$. If the system in (4.1) is consistent, then the solution is unique.

Proof. Assume that $X$ satisfies (4.1), that is $X=A^{D} A X$ and $A^{m} X=A^{m}\left(A^{j}\right)^{\dagger}$. Then $X=A^{D} A X=\left(A^{D}\right)^{m} A^{m} X=\left(A^{D}\right)^{m} A^{m}\left(A^{j}\right)^{\dagger}=A^{D} A\left(A^{j}\right)^{\dagger}$. Thus $X$ is unique.

By Theorem 9 if $X$ exists, then it is unique and denoted by $A_{j, m}^{\ominus}$.

Theorem 10. Let $A \in \mathbb{C}^{n \times n}$ and $m, j \in \mathbb{N}$.

(1) If $m \geq \operatorname{ind}(A)$, then the system in (4.1) is consistent and the solution is $X=$ $A^{D} A\left(A^{j}\right)^{\dagger}$. 
(2) If the system in (4.1) is consistent, then $\operatorname{ind}(A) \leq \max \{j, m\}$.

Proof. (1). Let $X=A^{D} A\left(A^{j}\right)^{\dagger}$. We have $A^{D} A X=A^{D} A A^{D} A\left(A^{j}\right)^{\dagger}=$ $A^{D} A\left(A^{j}\right)^{\dagger}=X$ and $A^{m} X=A^{m} A^{D} A\left(A^{j}\right)^{\dagger}=A^{D} A A^{m}\left(A^{j}\right)^{\dagger}=A^{m}\left(A^{j}\right)^{\dagger}$.

(2). If the system in (4.1) is consistent, then exits $X_{0} \in \mathbb{C}^{n \times n}$ such that $X_{0}=$ $A^{D} A X_{0}=\left(A^{D}\right)^{m} A^{m} X_{0}=\left(A^{D}\right)^{m} A^{m}\left(A^{j}\right)^{\dagger}=A^{D} A\left(A^{j}\right)^{\dagger}$ and $A^{m}\left(A^{j}\right)^{\dagger}=$ $A^{m} X_{0}=A^{m} A^{D} A\left(A^{j}\right)^{\dagger}=A^{m}\left(A^{D}\right)^{j} A^{j}\left(A^{j}\right)^{\dagger}$. Thus

$$
A^{m}\left(A^{j}\right)^{\dagger} A^{j}=A^{m}\left(A^{D}\right)^{j} A^{j}\left(A^{j}\right)^{\dagger} A^{j}=A^{m}\left(A^{D}\right)^{j} A^{j}=A^{m} A^{D} A .
$$

If $m \geq j$, then $A^{m} A^{D} A=A^{m}\left(A^{j}\right)^{\dagger} A^{j}=A^{m-j} A^{j}\left(A^{j}\right)^{\dagger} A^{j}=A^{m-j} A^{j}=A^{m}$. That is, $\operatorname{ind}(A) \leq m$. If $j>m$, then $A^{j}=A^{j}\left(A^{j}\right)^{\dagger} A^{j}=A^{j-m} A^{m}\left(A^{j}\right)^{\dagger} A^{j}=$ $A^{j-m} A^{m} A^{D} A=A^{j} A^{D} A$. That is, $\operatorname{ind}(A) \leq j$. Thus, $\operatorname{ind}(A) \leq \max \{j, m\}$.

Example 2. We will give an example that shows if $m<\operatorname{ind}(A)$, then the system in (4.1) is not consistent. Let $A$ be the same matrix in Example 1. It is easy to get $\operatorname{ind}(A)=2$ and $A^{D}=0$. Let $m=j=1$ and suppose that $X$ is the solution of system in (4.1), then $X=A^{D} A X=0$, which gives $A A^{\dagger}=A X=0$, thus $A=A A^{\dagger} A=0$, this is a contradiction.

Example 3. The converse of Theorem 10 (1) is not true. Let $m=1$ and $j=3$. If we let

$$
A=\left[\begin{array}{lll}
0 & 1 & 0 \\
0 & 0 & 1 \\
0 & 0 & 0
\end{array}\right],
$$

then $\operatorname{ind}(A)=3$ and $A^{3}=0$. Hence $X=0$ is a solution of (4.1), but $m<\operatorname{ind}(A)$.

Example 4. If ind $(A) \leq \max \{j, m\}$, then the system in (4.1) may be not consistent. If we let

$$
A=\left[\begin{array}{ccc}
2 & 2 & 1 \\
-1 & -1 & 0 \\
0 & 0 & 0
\end{array}\right] \text {, }
$$

then

$$
A^{3}=A^{2}=\left[\begin{array}{ccc}
2 & 2 & 2 \\
-1 & -1 & -1 \\
0 & 0 & 0
\end{array}\right] \text {, }
$$

$A^{D}=A^{2}$ and $\operatorname{ind}(A)=2$. Let $m=1$ and $j=2$, then $\operatorname{ind}(A) \leq \max \{j, m\}$. It is easy to check that

$$
\left(A^{2}\right)^{\dagger}=\frac{1}{15}\left[\begin{array}{lll}
2 & -1 & 0 \\
2 & -1 & 0 \\
2 & -1 & 0
\end{array}\right] \text {. }
$$


If the system in (4.1) has a solution $X_{0}$, then $X_{0}=A^{D} A X_{0}=A^{D} A\left(A^{2}\right)^{\dagger}$ and $A\left(A^{2}\right)^{\dagger}=A X_{0}=A A^{D} A\left(A^{2}\right)^{\dagger}=A^{4}\left(A^{2}\right)^{\dagger}=A^{2}\left(A^{2}\right)^{\dagger}$ would hold. But

$$
A\left(A^{2}\right)^{\dagger}=\frac{1}{15}\left[\begin{array}{ccc}
10 & -5 & 0 \\
-4 & 2 & 0 \\
0 & 0 & 0
\end{array}\right] \neq \frac{1}{15}\left[\begin{array}{ccc}
12 & -6 & 0 \\
-6 & 3 & 0 \\
0 & 0 & 0
\end{array}\right]=A^{2}\left(A^{2}\right)^{\dagger} .
$$

Thus, the system in (4.1) is not consistent.

Remark 8. If $m \geq \operatorname{ind}(A)=k$, it is not difficult to see that $A_{j, m}^{\ominus}=A_{j, m+1}^{\ominus}$. That is to say, the $(j, m)$-core inverse of $A$ coincides with the $(j, m+1)$-core inverse of $A$. Thus, in the sequel, we only discuss the $m=\operatorname{ind}(A)$ case.

Theorem 11. Let $A, X \in \mathbb{C}^{n \times n}, k, j \in \mathbb{N}$. If $\operatorname{ind}(A)=k$ and $X$ is the $(j, k)$-core inverse of $A$, then we have $X^{j} A^{j} X^{j}=\left(A^{D}\right)^{j(j-1)} X^{j}$ and $X A^{j}=A^{D} A$.

Proof. By the definition of the $(j, k)$-core inverse, we have $X=A^{D} A X$ and $A^{k} X=A^{k}\left(A^{j}\right)^{\dagger}$. By $X=A^{D} A\left(A^{j}\right)^{\dagger}$, it is easy to check that $X^{n+1}=\left(A^{D}\right)^{j} X^{n}$ for arbitrary $n \in \mathbb{N}$, which gives that $X^{j}=\left(A^{D}\right)^{j(j-1)} X$.

$$
\begin{aligned}
X A^{j} & =A^{D} A\left(A^{j}\right)^{\dagger} A^{j}=\left(A^{D}\right)^{j} A^{j}\left(A^{j}\right)^{\dagger} A^{j}=\left(A^{D}\right)^{j} A^{j}=A^{D} A ; \\
X^{j} A^{j} X^{j} & =\left(A^{D}\right)^{j(j-1)} X A^{j} X^{j}=\left(A^{D}\right)^{j(j-1)} A^{D} A X^{j}=\left(A^{D}\right)^{j(j-1)} X^{j} .
\end{aligned}
$$

Corollary 2. Let $A, X \in \mathbb{C}^{n \times n}$ and $\operatorname{ind}(A)=k$. If $X$ is the $(1, k)$-core inverse of $A$, then we have $X A X=X$ and $X A=A^{D} A$.

The $(j, m)$-core inverse is a generalization of the core inverse and the DMP-inverse in view of Theorem 11.

Remark 9. When $j=m=1=\operatorname{ind}(A)$, the equations in (4.1) are equivalent to $X A X=X, X A=A^{\#} A$, and $A X=A A^{\dagger}$. Thus $A X=A A^{\dagger}$ implies that $(A X)^{*}=$ $A X ; X A=A^{\#} A$ gives that $X A^{2}=A$ and $A X A=A$; and $X=X A X=A^{\#} A X=$ $A A^{\#} X$, which means that $\mathcal{R}(X) \subseteq \mathcal{R}(A)$, then $X=A Y$ for some $Y \in \mathbb{C}^{n \times n}$, thus $X=A Y=A X A Y=A X^{2}$. Therefore, we have $A^{\boxplus}=X$ by Lemma 1. In a word, the $(1,1)$-core inverse coincides with the usual core inverse.

Remark 10. If we let $j=1$ and $m=\operatorname{ind}(A)$, then the equations in (4.1) are equivalent to $X A X=X, X A=A^{D} A$, and $A^{k} X=A^{k} A^{\dagger}$ by Theorem 11 . Thus $(1, k)$-core inverse coincides with the DMP-inverse.

From Remark 10, Theorem 11 and the definition of the $(j, k)$-core inverse, we have the following theorem, which says that the conditions $X A X=X$, and $X A=A^{D} A$ in the definition of the DMP-inverse can be replaced by $X=A^{D} A X$.

Theorem 12. Let $A \in \mathbb{C}^{n \times n}$ with $k=\operatorname{ind}(A)$. Then $X \in \mathbb{C}^{n \times n}$ is the DMP-inverse of $A$ if and only if $X=A^{D} A X$ and $A^{k} X=A^{k} A^{\dagger}$. 
In the following theorem, we will give a canonical form for the $(j, k)$-core inverse of a matrix $A \in \mathbb{C}^{n \times n}$ by using the matrix decomposition in Theorem 1 .

Theorem 13. Let $A \in \mathbb{C}^{n \times n}$ have the form (2.1) with $\operatorname{ind}(A)=k$ and $j \in \mathbb{N}$. Then

$$
A_{j, k}^{\ominus}=U\left[\begin{array}{cc}
(M C)^{D}(M C)_{j-1, k}^{\ominus} & 0 \\
0 & 0
\end{array}\right] U^{*} .
$$

Proof. By Theorem 10 and the idempotency of $A^{D} A$ we have

$$
A_{j, k}^{\ominus}=A^{D} A\left(A^{j}\right)^{\dagger}=\left(A^{D}\right)^{j} A^{j}\left(A^{j}\right)^{\dagger} .
$$

From the proof of Theorem 7, we have

$$
A^{j}\left(A^{j}\right)^{\dagger}=U\left[\begin{array}{cc}
(M C)^{j-1}\left((M C)^{j-1}\right)^{\dagger} & 0 \\
0 & 0
\end{array}\right] U^{*} .
$$

By (2.2) we have

$$
\left(A^{D}\right)^{j}=U\left[\begin{array}{cc}
{\left[(M C)^{D}\right]^{j}} & {\left[(M C)^{D}\right]^{j+1} M S} \\
0 & 0
\end{array}\right] U^{*} .
$$

By the proof of Theorem 7, we have ind $(M C) \leq k-1<k$. From (4.2), (4.3) and (4.4), we have

$$
\begin{aligned}
A_{j, k}^{\ominus} & =\left(A^{D}\right)^{j} A^{j}\left(A^{j}\right)^{\dagger} \\
& =U\left[\begin{array}{cc}
{\left[(M C)^{D}\right]^{j}} & {\left[(M C)^{D}\right]^{j+1} M S} \\
0 & 0
\end{array}\right]\left[\begin{array}{cc}
(M C)^{j-1}\left((M C)^{j-1}\right)^{\dagger} & 0 \\
0 & 0
\end{array}\right] U^{*} \\
& =U\left[\begin{array}{cc}
{\left[(M C)^{D}\right]^{j}(M C)^{j-1}\left((M C)^{j-1}\right)^{\dagger}} & 0 \\
0 & 0
\end{array}\right] U^{*} \\
& =U\left[\begin{array}{cc}
(M C)^{D}\left[(M C)^{D}\right]^{j-1}(M C)^{j-1}\left((M C)^{j-1}\right)^{\dagger} & 0 \\
0 & 0
\end{array}\right] U^{*} \\
& =U\left[\begin{array}{cc}
(M C)^{D}(M C)^{D} M C\left((M C)^{j-1}\right)^{\dagger} & 0 \\
0 & 0
\end{array}\right] U^{*} \\
& =U\left[\begin{array}{cc}
(M C)^{D}(M C)_{j-1, k}^{\ominus} & 0 \\
0 & 0
\end{array}\right] U^{*} .
\end{aligned}
$$

Remark 11. If we use the decomposition of Hartwig and Spindelböck in [8, Corollary 6], then an expression of the $(j, k)$-core inverse of $A$ is

$$
A_{j, k}^{\ominus}=U\left[\begin{array}{cc}
(\Sigma K)^{D}(\Sigma K)_{j-1, k}^{\ominus} & 0 \\
0 & 0
\end{array}\right] U^{*},
$$

which is similar to the expression of $A_{j, k}^{\ominus}$ in Theorem 13. Since the proof of this result can be proved like the proof of Theorem 13, we omit this proof. 
Theorem 14. Let $A \in \mathbb{C}^{n \times n}$ and $\operatorname{ind}(A)=k$. If $\left(A^{k} X^{k}\right)^{*}=A^{k} X^{k}, A X^{k+1}=$ $X^{k}$ and $X A^{k+1}=A^{k}$, then $A$ is $(k, k)$-core invertible and $A_{k, k}^{\ominus}=X^{k}$.

Proof. By Lemma 2 and Lemma 3, we have $A^{k} X^{k} A^{k}=A^{k}, X^{k} A^{k} X^{k}=X^{k}$, $A^{k}=X^{k} A^{2 k}$, and $A^{D}=X^{k+1} A^{k}$. Equalities $\left(A^{k} X^{k}\right)^{*}=A^{k} X^{k}$ and $A^{k} X^{k} A^{k}=$ $A^{k}$ imply that $X^{k}$ is a $\{1,3\}$-inverse of $A^{k}$. From $A^{D}=X^{k+1} A^{k}$, we can obtain $\left(A^{D}\right)^{k}=X^{k-1} A^{D}$ by induction. Thus

$$
\begin{aligned}
A_{k, k}^{\ominus} & =A^{D} A\left(A^{k}\right)^{\dagger}=\left(A^{D}\right)^{k} A^{k}\left(A^{k}\right)^{\dagger}=\left(A^{D}\right)^{k} A^{k}\left(A^{k}\right)^{(1,3)} \\
& =\left(A^{D}\right)^{k} A^{k} X^{k}=\left(X^{k+1} A^{k}\right)^{k} A^{k} X^{k}=X^{k-1} X^{k+1} A^{k} A^{k} X^{k} \\
& =X^{2 k} A^{2 k} X^{k}=X^{k}\left(X^{k} A^{2 k}\right) X^{k}=X^{k} A^{k} X^{k}=X^{k} .
\end{aligned}
$$

Proposition 3. Let $A \in \mathbb{C}^{n \times n}$ be a matrix with $j \geq \operatorname{ind}(A)=k$. If $A$ is $(j, k)$-core invertible, then $A^{j} A_{j, k}^{\ominus}$ is the projector onto $\mathcal{R}\left(A^{j}\right)$ along $\mathcal{R}\left(A^{j}\right)^{\perp}$.

Proof. It is trivial.

In the following proposition, we will investigate some properties of the $(j, k)$-core inverse.

Proposition 4. Let $A \in \mathbb{C}^{n \times n}$ with $j \geq \operatorname{ind}(A)=k$. If $A$ is $(j, k)$-core invertible, then

(1) $A_{j, k}^{\ominus}$ is a $\{1,2,3\}$-inverse of $A^{j}$;

(2) $A_{j, k}^{\ominus}=\left(A^{D}\right)^{j} P_{A^{j}}$;

(3) $\left(A_{j, k}^{\ominus}\right)^{n}= \begin{cases}{\left[\left(A^{D}\right)^{j}\left(A^{j}\right)^{\dagger}\right]^{n / 2}} & \text { if } n \text { is even, } \\ A^{j}\left[\left(A^{D}\right)^{j}\left(A^{j}\right)^{\dagger}\right]^{(n+1) / 2} & \text { if } n \text { is odd. }\end{cases}$

(4) $A_{j, k}^{\ominus} A^{D}=\left(A^{D}\right)^{j+1}$;

(5) $A^{j} A_{j, k}^{\ominus}=A_{j, k}^{\ominus} A^{j}$ if and only if $\mathcal{R}\left(A^{j}\right)^{\perp} \subseteq \mathcal{N}\left(A^{j}\right)$;

(6) $A_{j, k}^{\ominus}=A$ implies that $A$ is EP.

Proof. (1). By Theorem 10 we have $A_{j, k}^{\ominus}=A^{D} A\left(A^{j}\right)^{\dagger}=\left(A^{D}\right)^{j} A^{j}\left(A^{j}\right)^{\dagger}$, thus

$$
\begin{aligned}
A^{j} A_{j, k}^{\ominus} A^{j} & =A^{j}\left(A^{D}\right)^{j} A^{j}\left(A^{j}\right)^{\dagger} A^{j}=A^{j}\left(A^{D}\right)^{j} A^{j}=A^{j} A^{D} A=A^{j} ; \\
A_{j, k}^{\ominus} A^{j} A_{j, k}^{\ominus} & =\left(A^{D}\right)^{j} A^{j}\left(A^{j}\right)^{\dagger} A^{j} A_{j, k}^{\ominus}=A^{D} A A_{j, k}^{\ominus} \\
& =A^{D} A A^{D} A\left(A^{j}\right)^{\dagger}=A^{D} A\left(A^{j}\right)^{\dagger}=A_{j, k}^{\ominus} ; \\
A^{j} A_{j, k}^{\ominus} & =A^{j}\left(A^{D}\right)^{j} A^{j}\left(A^{j}\right)^{\dagger}=A^{j}\left(A^{j}\right)^{\dagger} .
\end{aligned}
$$

(2) is trivial. 
(3). By $\left(A_{j, k}^{\ominus}\right)^{2}=\left(A^{D}\right)^{j} A^{j}\left(A^{j}\right)^{\dagger}\left(A^{D}\right)^{j} A^{j}\left(A^{j}\right)^{\dagger}=\left(A^{D}\right)^{j}\left(A^{j}\right)^{\dagger}$ and induction it is easy to check (3).

(4). $A_{j, k}^{\ominus} A^{D}=\left(A^{D}\right)^{j} A^{j}\left(A^{j}\right)^{\dagger} A^{D}=\left(A^{D}\right)^{j} A^{j}\left(A^{j}\right)^{\dagger}\left(A^{D}\right)^{j} A^{j} A^{D}=\left(A^{D}\right)^{j+1}$.

(5). By $\mathcal{R}\left[I_{n}-A^{j}\left(A^{j}\right)^{\dagger}\right]=\mathcal{N}\left[\left(A^{j}\right)^{\dagger}\right]$ and $\mathcal{N}\left(A^{D} A\right)=\mathcal{N}\left(A^{D}\right)$, we have

$$
\begin{aligned}
A^{j} A_{j, k}^{\ominus}=A_{j, k}^{\ominus} A^{j} & \Leftrightarrow A^{j}\left(A^{D}\right)^{j} A^{j}\left(A^{j}\right)^{\dagger}=\left(A^{D}\right)^{j} A^{j}\left(A^{j}\right)^{\dagger} A^{j} \\
& \Leftrightarrow A^{j}\left(A^{D}\right)^{j} A^{j}\left(A^{j}\right)^{\dagger}=\left(A^{D}\right)^{j} A^{j} \\
& \Leftrightarrow A^{j}\left(A^{D}\right)^{j}\left[I_{n}-A^{j}\left(A^{j}\right)^{\dagger}\right]=0 \\
& \Leftrightarrow \mathcal{R}\left[I_{n}-A^{j}\left(A^{j}\right)^{\dagger}\right] \subseteq \mathcal{N}\left(A^{D} A\right) \\
& \Leftrightarrow \mathcal{N}\left[\left(A^{j}\right)^{\dagger}\right] \subseteq \mathcal{N}\left(A^{D} A\right) \\
& \Leftrightarrow \mathcal{N}\left[\left(A^{j}\right)^{*}\right] \subseteq \mathcal{N}\left(A^{D}\right) \\
& \Leftrightarrow \mathcal{R}\left(A^{j}\right)^{\perp} \subseteq \mathcal{N}\left(A^{j}\right) .
\end{aligned}
$$

(6). Let $A$ be written in the form (2.1). We have

$$
A_{j, k}^{\ominus}=U\left[\begin{array}{cc}
(M C)^{D}(M C)_{j-1, k}^{\ominus} & 0 \\
0 & 0
\end{array}\right] U^{*}
$$

by Theorem 13. Thus, $A_{j, k}^{\ominus}=A$ implies $M S=0$. From the nonsingularity of $M$, we have $S=0$, which is equivalent to say that $A$ is EP in view of [2, Theorem 3.7].

In the following proposition, we shall give the the relationship between the $(j, k)$ core inverse and DMP-inverse and core-EP inverse.

Proposition 5. Let $A \in \mathbb{C}^{n \times n}$ with $\operatorname{ind}(A)=k$. Then

$$
A_{k, k}^{\ominus}=A^{D, \dagger}\left(A^{D}\right)^{k-1} A A^{\oplus} .
$$

Proof. We have that $A^{k}\left(A^{k}\right)^{\dagger}=A A^{\oplus}$ by Lemma 5 and $A^{D, \dagger}=A^{D} A A^{\dagger}$. Thus

$$
\begin{aligned}
A_{k, k}^{\ominus} & =A^{D} A\left(A^{k}\right)^{\dagger}=\left(A^{D}\right)^{k} A^{k}\left(A^{k}\right)^{\dagger}=A^{D} A^{k}\left(A^{D}\right)^{k-1}\left(A^{k}\right)^{\dagger} \\
& =A^{D} A A^{\dagger} A^{k}\left(A^{D}\right)^{k-1}\left(A^{k}\right)^{\dagger}=A^{D, \dagger}\left(A^{D}\right)^{k-1} A^{k}\left(A^{k}\right)^{\dagger} \\
& =A^{D, \dagger}\left(A^{D}\right)^{k-1} A A^{\oplus} .
\end{aligned}
$$

In the following theorem, we will give a relationship between the $\langle i, m\rangle$-core inverse and $(j, m)$-core inverse.

Theorem 15. Let $A \in \mathbb{C}^{n \times n}$ with $\operatorname{ind}(A)=k$. Then $A_{k, m}^{\oplus}=A_{m, k}^{\ominus}$ for any $m \geq k$. 
Proof. By Theorem 10, we have $A_{m, k}^{\ominus}=A^{D} A\left(A^{m}\right)^{\dagger}=\left(A^{D}\right)^{k} A^{k}\left(A^{m}\right)^{\dagger}$. By the proof of Remark 5, we have $A^{k}=M N$ and $N M=L^{k}$, where $M=\prod_{l=1}^{k} B_{l}$, $N=\prod_{l=1}^{k} G_{k+1-l}$ and $L=G_{k} B_{k}$. It is easy to see that $\left(A^{D}\right)^{s}=M L^{-k-s} N$ for any $s \in \mathbb{N}$ by $N M=L^{k}$. Thus $\left(A^{D}\right)^{k}=M L^{-2 k} N$ and

$$
\left(A^{D}\right)^{k} A^{k}=M L^{-2 k} N M N=M L^{-2 k} L^{k} N=M L^{-k} N .
$$

By the proof of Remark 5, we have $A^{m}=M L^{m-k} N=M_{1} N$ is a full rank factorization of $A^{m}$, where $M_{1}=M L^{m-k}$ and

$$
\left(A^{m}\right)^{\dagger}=N^{*}\left(N N^{*}\right)^{-1}\left(M_{1}^{*} M_{1}\right)^{-1}\left(M_{1}\right)^{*} .
$$

By Theorem 6, we have $A_{k, m}^{\oplus}=M L^{-m} M^{\dagger}$. In the following steps, we will show that $A_{m, k}^{\ominus}=M L^{-m} M^{\dagger}$. From $A_{m, k}^{\ominus}=\left(A^{D}\right)^{k} A^{k}\left(A^{m}\right)^{\dagger}$, we have

$$
\begin{aligned}
A_{k, m}^{\ominus} & =\left(A^{D}\right)^{k} A^{k}\left(A^{m}\right)^{\dagger}=M L^{-k} N N^{*}\left(N N^{*}\right)^{-1}\left(M_{1}^{*} M_{1}\right)^{-1}\left(M_{1}\right)^{*} \\
& =M L^{-k}\left(M_{1}^{*} M_{1}\right)^{-1}\left(M_{1}\right)^{*}=M L^{-k}\left[\left(L^{m-k}\right)^{*} M^{*} M L^{m-k}\right]^{-1}\left(L^{m-k}\right)^{*} M^{*} \\
& =M L^{-k} L^{k-m}\left(M^{*} M\right)^{-1}\left[\left(L^{m-k}\right)^{*}\right]^{-1}\left(L^{m-k}\right)^{*} M^{*} \\
& =M L^{-m}\left(M^{*} M\right)^{-1} M^{*}=M L^{-m} M^{\dagger} .
\end{aligned}
$$

Theorem 16. Let $A \in \mathbb{C}^{n \times n}$ with $i \geq \operatorname{ind}(A)=k$, then $A_{i, k}^{\ominus}=P_{1} D^{-i} P_{1}^{\dagger}$, where $A=P\left[\begin{array}{cc}D & 0 \\ 0 & N\end{array}\right] P^{-1}$ with $D \in \mathbb{C}^{r \times r}$ is nonsingular, $N$ is nilpotent and $P=\left[P_{1} \mid P_{2}\right]$ with $P_{1} \in \mathbb{C}^{n \times r}$.

Proof. It is easy to see that by Theorem 8 and Theorem 15 .

\section{ACKNOWLEDGEMENT}

This research is supported by the National Natural Science Foundation of China (NO.11771076 and No. 11471186). The first author is grateful to China Scholarship Council for giving him a purse for his further study in Universitat Politècnica de València, Spain.

\section{REFERENCES}

[1] O. M. Baksalary and G. Trenkler, "Core inverse of matrices," Linear Multilinear Algebra, vol. 58, no. 6, pp. 681-697, 2010, doi: 10.1080/03081080902778222.

[2] J. Benítez, "A new decomposition for square matrices," Electron. J. Linear Algebra, vol. 20, pp. 207-225, 2010, doi: 10.13001/1081-3810.1369.

[3] J. Benítez and X. Liu, "A short proof of a matrix decomposition with applications," Linear Algebra Appl., vol. 438, no. 3, pp. 1398-1414, 2013, doi: 10.1016/j.laa.2012.10.002.

[4] S. L. Campbell and C. D. Meyer, Generalized Inverses of Linear Transformations. Philadelphia, SIAM, 2009. 
[5] J. Chen, "Group inverses and drazin inverses of matrices over rings, (chinese)," J. Xinjiang Univ. Natur. Sci., vol. 9, no. 1, pp. 44-49, 1992.

[6] R. E. Cline, "An application of representations for the generalized inverse of a matrix," Mathematics Research Center, University of Wisconsin, Madison, Tech. Rep., 1965.

[7] R. E. Cline, "Inverses of rank invariant powers of a matrix," SIAM J. Numer. Anal., vol. 5, no. 1, pp. 182-197, 1968, doi: 10.1137/0705015.

[8] R. E. Hartwig and K. Spindelböck, "Matrices for which $a^{*}$ and $a^{\dagger}$ commute," Linear Multilinear Algebra, vol. 14, no. 3, pp. 241-256, 1983, doi: 10.1080/03081088308817561.

[9] S. B. Malik and N. Thome, "On a new generalized inverse for matrices of an arbitrary index," Appl. Math. Comput., vol. 226, pp. 575-580, 2014, doi: doi.org/10.1016/j.amc.2013.10.060.

[10] K. Manjunatha Prasad and K. S. Mohana, "Core-ep inverse," Linear Multilinear Algebra, vol. 62, no. 6, pp. 792-802, 2014, doi: 10.1080/03081087.2013.791690.

[11] H. Wang, "Core-ep decomposition and its applications," Linear Algebra Appl., vol. 508, pp. 289300, 2016, doi: 10.1016/j.laa.2016.08.008.

[12] H. K. Wimmer, "Canonical angles of unitary spaces and perturbations of direct complements," Linear Algebra Appl., vol. 287, pp. 373-379, 1999, doi: 10.1016/S0024-3795(98)10017-4.

[13] S. Xu, J. Chen, and X. Zhang, "New characterizations for core inverses in rings with involution," Front. Math. China, vol. 12, no. 1, pp. 231-246, 2017, doi: 10.1007/s11464-016-0591-2.

Authors' addresses

\section{Sanzhang Xu}

Faculty of Mathematics and Physics, Huaiyin Institute of Technology, 223003 Huaian, China

E-mail address: xusanzhang52220126.com

\section{Jianlong Chen}

School of Mathematics, Southeast University, 210096 Nanjing, China

E-mail address: E-mail: jlchen@seu.edu.cn

\section{Julio Benítez}

Universitat Politècnica de València, Instituto de Matemática Multidisciplinar, 46022 Valencia, Spain

E-mail address: jbenitez@mat.upv.es

\section{Dingguo Wang}

School of Mathematical Sciences, Qufu Normal University, 273165 Qufu, China

E-mail address: dingguo95@126.com 